\title{
Carlos Liscano : les listes comme réservoir de projets
}

\section{Fatiha Idmhand}

\section{(2) OpenEdition}

1 Journals

\section{Édition électronique}

URL : http://journals.openedition.org/genesis/3677

DOI : 10.4000/genesis.3677

ISSN : 2268-1590

\section{Éditeur :}

Presses universitaires de Paris Sorbonne (PUPS), Société internationale de génétique artistique littéraire et scientifique (SIGALES)

\section{Édition imprimée}

Date de publication : 15 décembre 2018

Pagination : 93-103

ISBN : 979-10-231-06282

ISSN : 1167-5101

\section{Référence électronique}

Fatiha Idmhand, «Carlos Liscano : les listes comme réservoir de projets », Genesis [En ligne], 47 | 2018, mis en ligne le 15 décembre 2019, consulté le 21 janvier 2021. URL : http://journals.openedition.org/ genesis/3677 ; DOI : https://doi.org/10.4000/genesis.3677 


\title{
Carlos Liscano : les listes comme réservoirs de projets
}

\author{
Fatiha Idmhand
}

We should end the practice of viewing words as crimes*.

PEN International.

L es manuscrits de prison des écrivains constituent des corpus rares auxquels il est difficile d'accéder à cause des raisons de l'incarcération de leurs auteurs, des conditions contextuelles et matérielles de leur production et des règles légales ${ }^{1}$, souvent très strictes, qui peuvent régir la sortie de tels écrits hors de la prison ${ }^{2}$. Dans le champ de la littérature latino-américaine produite durant les années 1970, années des dictatures les plus violentes de l'histoire de ce continent, de tels manuscrits sont très exceptionnels. C'est presque par miracle que Carlos Liscano (Montevideo, 1949-) a pu sauver les siens des geôles uruguayennes ${ }^{3}$ : ils racontent, depuis l'intérieur, les formes de la répression durant les «guerres sales » du Cône Sud et ce que fut la vie des intellectuels détenus par les militaires ${ }^{4}$. Mais, surtout, ils sont les témoins de la genèse carcérale d'une vocation pour l'écriture.

Dans ces brouillons que Carlos Liscano consulte encore, plus de trente ans après leur composition, on trouve de nombreuses listes qui approvisionnent toujours les projets de l'écrivain. Comment définir le statut particulier occupé par ces listes «provisionnelles » qui nourrissent, non pas un projet d'écriture, mais tous les projets d'écriture de la vie de l'écrivain? L'étude croisée de la genèse de ces listes et de leur devenir hors du pénitencier vise à apporter des éléments de réponse à cette question.

\section{Écrire en prison}

L'entrée en écriture de Carlos Liscano est le résultat d'un ensemble d'éléments factuels. Arrêté à l'âge de vingt-trois ans le 27 mai 1972 par la police militaire uruguayenne, il en aura trente-six lorsqu'il quittera enfin sa cellule le 15 mars 1985. Issu d'une famille ouvrière de la banlieue de Montevideo (La Teja) qui ne militait pas et vivait loin des cercles intellectuels et politiques de l'époque, il s'engage très tôt dans l'armée pour obtenir une petite autonomie financière. Élève du Liceo Militar, il intègre ensuite la Escuela Militar de Aeronáutica dont il est exclu en 1970, à cause de propos critiques à l'encontre des décisions politiques du président Pacheco 5 et pour avoir dénoncé la corruption au sein de la Fuerza Aérea ${ }^{6}$. Dès 1970, il connaît donc une première et courte détention politique 7 qui débouche sur son renvoi de l'armée et de la Escuela Militar. C'est alors qu'il rejoindra le Movimiento de Liberación Nacional Tupamaro

(*) PEN, Chair's Notebook \#2, 2009, consultable en ligne.

1. En France par exemple : «La sortie des écrits faits par un détenu en vue de leur publication ou de leur divulgation sous quelque forme que ce soit est autorisée par décision du directeur interrégional des services pénitentiaires territorialement compétent. » Consultable en ligne.

2. Jean-Pierre Cavaillé, Cahiers du Centre de recherches historiques $\mathrm{n}^{\circ}$ 39, «Écriture et prison sous l'Ancien Régime», EHESS, avril 2007, p. $10-11$.

3. En 1992, dans l'épigraphe de la première édition de La Mansión del tirano, Carlos Liscano raconte le miraculeux sauvetage de ses manuscrits, sortis de la prison dans la guitarre de Heber Esquivo. Voir Carlos Liscano, La Mansión del tirano, Montevideo, Arca, 1992, p. 184-185.

4. Les carnets de l'Argentine Alicia Kozameh (Rosario, 1953-), écrits durant sa détention à la Villa Devoto entre 1975 et 1978, numérisés en 2011 par le Centre de recherche latino-américain de Poitiers et consultables en ligne, présentent des analogies avec les manuscrits de Carlos Liscano. Voir les travaux d'Amandine Guillard consultables en ligne : «Poétique du corps enfermé. Répression et résistance des prisonnières politiques argentines (1976-1983)», Amerika, n 11, 2014; « Resistencia y poesía en las cárceles argentinas (1976-1983)», Amerika, $\mathrm{n}^{\circ}$ 8, 2013. Nous renvoyons également à notre ouvrage à paraître en 2019 : Fatiha Idmhand, Carlos Liscano : de la contrainte à l'œuvre, aux Éditions des Archives contemporaines.

5. Voir Carlos Demasi, Jorge Landinelli, María Sara López, Álvaro Rico, La Caída de la democracia. Cronología comparada de la historia reciente del Uruguay (1967-1973), Montevideo, Fundación cultura universitaria, 1996.

6. Clara Aldrighi, Memorias de insurgencia : historias de vida y militancia en el MLN-Tupamaros 1965-1975, Montevideo, Ediciones de la Banda Oriental, 2009.

7. Ibid. 
$(M L N \text {-Tupamaro })^{8}$ et ses actions directes. Au cours de l'une d'elles, en 1972, il est arrêté avec les dirigeants du mouvement $M L N$. Comme eux, il passera treize ans en prison. Toutefois, n'étant ni fondateur ni dirigeant du $M L N$, il parvient à éviter la réclusion permanente au cachot. Il n'y séjourne que pour y être torturé; le reste du temps, il est avec d'autres, dans une cellule du pénitencier du village de Libertad.

Contrairement à d'autres membres du $M L N$ qui furent emprisonnés au même moment que lui, comme Mauricio Rosencof (Uruguay, 1933-) par exemple, Carlos Liscano n'avait reçu aucune instruction lettrée, ni publié avant sa détention : c'est bien en prison qu'il se forme à l'écriture et qu'il compose ses premières et plus importantes œuvres littéraires ${ }^{9}$. Le corpus carcéral comporte 872 pages écrites entre 1981 et 1984 : il s'agit des brouillons des œuvres les plus importantes de sa production ${ }^{10}$. Les 476 feuillets offrent presque tous la même présentation (voir fig. 1) : une même couleur jaune paille et des pages saturées d'écriture qui laissent peu de marge ou d'espace entre les lignes et qui furent composées sur le papier (140-170 mm × 230-210 mm de long) que les militaires remettaient aux détenus pour la rédaction des correspondances à la famille11.

C'est lors de l'un de ses séjours dans le cachot appelé «La Isla» [L'île] ${ }^{12}$, après une session de torture, que Carlos Liscano décide de devenir écrivain et, comme il le dit souvent dans ses interviews et conférences, d' «inventer»sa figure d'auteur ${ }^{13}$.

Je sais exactement où et comment j'ai commencé à écrire. Je sais quelle est la première chose que j'ai écrite avec une intention littéraire. Je me souviens de la date : 1er février $19811^{14}$.

Le champ lexical et sémantique de la maladie lui permet d'expliquer le moment où il a entrevu, pour la première fois, son projet littéraire ${ }^{15}$. Toutefois, ses manuscrits, et notamment ses notes de lectures, montrent que cette littérature, qui semble être née du néant, n'est pas le fruit d'un si grand vide.

\section{La liste comme bibliothèque}

Comme l'a montré Alfredo Alzugarat ${ }^{16}$, la bibliothèque du Penal Libertad fut l'une des plus riches de l'époque : à la fois quantitativement, avec près de neuf mille livres recensés en 1985, et qualitativement, en raison du caractère exceptionnel de ses détenus. La bibliothèque a fonctionné de façon très régulière de 1973 à 1985 et fut soumise aux aléas de la censure, de la politique pénitentiaire, des durcissements ou infléchissements du régime. Enrichie des donations des familles et des amis des prisonniers, elle était gérée par les détenus, sous la surveillance des militaires, et était accessible aux prisonniers par le biais de fiches de prêt sur lesquelles ils reportaient une liste de numéros d'ouvrages,

8. Voir Alain Labrousse, Les Tupamaros. Des armes aux urnes, Paris, Éditions du Rocher, 2009, p. 80.

9. Son parcours rappelle celui de 1'Espagnol Fernando Macarro Castillo (San Vicente, Alconada, 1920 - Madrid, 2016), prisonnier du régime franquiste de 1939 à 1961 et devenu poète en prison, au milieu des années 1950 .

10. El Informante [Le rapporteur], La Mansión del tirano [La Maison du tyran], El Método y otros juguetes carcelarios [La Méthode et autres jeux captifs], El Diario de la cárcel [Le Journal de prison], El Juego de la letra cambiada [Le Jeu de la lettre troquée], Los Siete Mensajeros [Les Sept Messagers] et Apuntes de la cárcel [Notes de prison].

11. La lettre était la seule forme d'écriture autorisée. Sa rédaction était régie par des règles strictes : une seule lettre par semaine, aucun changement de couleur n'était autorisé, la présentation de la lettre devait être uniforme et les noms et adresses précises des correspondants clairement indiqués. Alfredo Alzugarat, Trincheras de papel. Dictadura y literatura carcelaria en Uruguay, Montevideo, Trilce, 2007, p. 11.

12. La Isla était le lieu où les militaires isolaient des prisonniers dans le but de les torturer. Voir El Furgón de los locos, Buenos Aires, Planeta, 2007 ; la version française, traduite par Jean-Marie Saint-Lu a été publiée en 2008 : Le Fourgon des fous, Paris, Éditions 10/18, 2008.

13. Voir Carlos Liscano, «Del caos a la literatura», dans Trazas y Ficciones. Literatura y psicoanálisis, Asociación Psicoanalítica del Uruguay, Biblioteca uruguaya de psicoanálisis, vol. VII, Montevideo, novembre 2007.

14. Carlos Liscano, La Mansión del tirano, Montevideo, Arca, 1992, p. 234.

15. Selon María Carolina Blixen, il ne s'agit pas d'un témoignage isolé puisque de nombreux détenus, notamment ceux qui ont été otages du régime, ont indiqué avoir vécu, dans les cachots, des phases d'aliénation ou de «délire» provoquées par les châtiments auxquels ils ont été soumis, et indiquent avoir frôlé la «folie». Les mêmes signalent avoir utilisé différentes «armes» telles que la religion, l'art ou la littérature pour se ressaisir et ne pas sombrer dans la folie. Voir María Carolina Blixen, «Figuras de la poética de Carlos Liscano : cuando la literatura es el surco del delirio. Ficción, autoficción, testimonio », thèse soutenue le 20 novembre 2013 à l'université Charles de Gaulle-Lille III, 2013, p. $57-58$.

16. Alfredo Alzugarat, Trincheras de papel, op. cit., p. 18-45. 


\section{"LA EDAD DR IA RRDSA"}

$$
\begin{aligned}
& \text { JE GRTAR SOLAMBNTG GUIERO DARTARMB. } \\
& \text { quEVIO. }
\end{aligned}
$$

-.. y ésa es le úinica historia, b pre yo desería conder, y no lo pere diga gan,

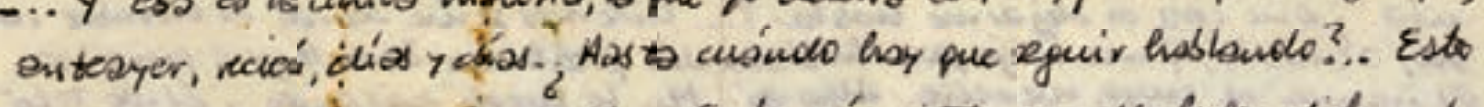

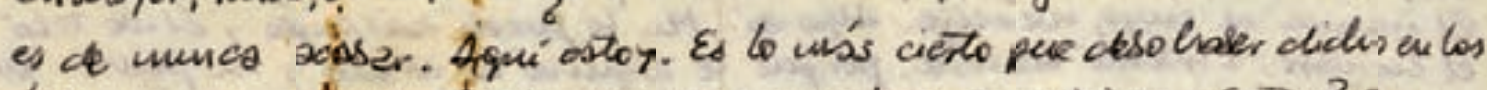

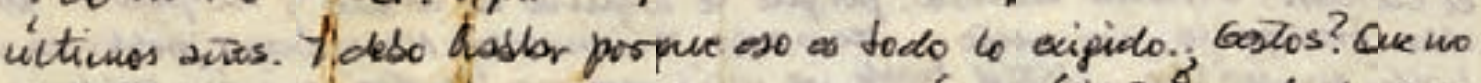

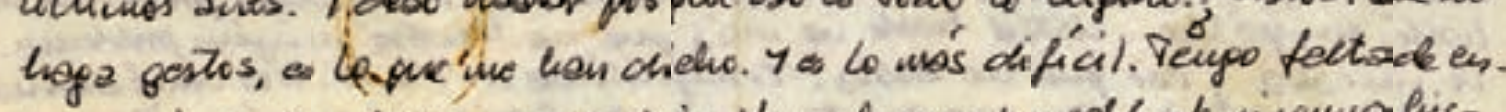

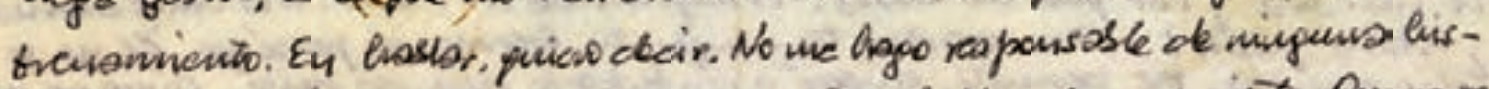
toria. Si en afgin monanto dijo pue no estoy hastaudo no do ciéto. Que no se

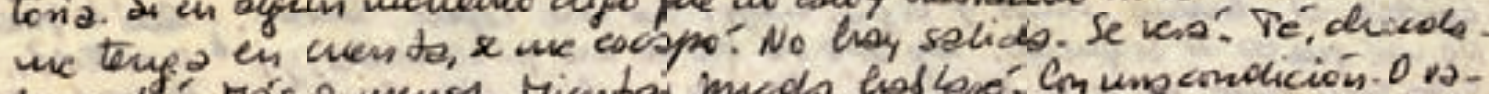

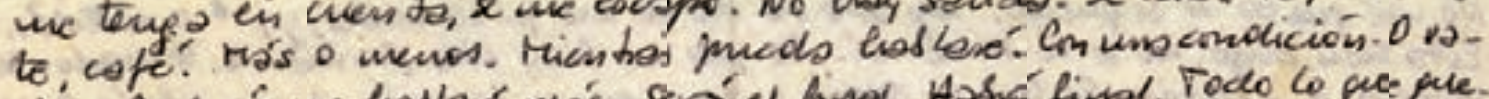
vis. Deopués uo lasshad más. Erá a find. Hassé find. Todo 40 pe pue. de será territosio de wadie. Operación tem tovio. Li una concosión. des mós zo vol vené al estado inicin, dide hace un rato, thes semanas, wo se?

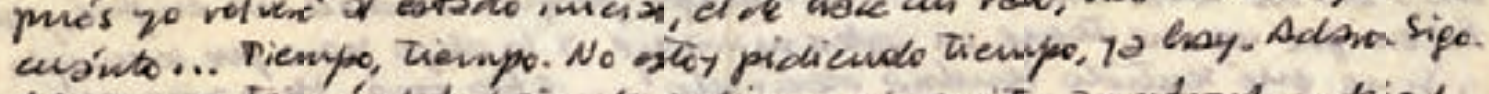
sfuien se tomó el tros gjo de susirue el carrito, and tranto y dejach equi. ' Y shord? Que hable. Eso es. Usted hodk. No me movere a del co. rrito por wads del numdo. Ni sipuiers para denme exe baño que me

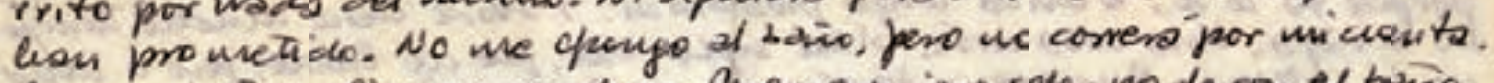
Que use beñen. Si de es su desee. Pero no puieso seber nods an el biño. En eso zons coroze de iniciativas. Estoy en defós sido iquá? .... Nade.

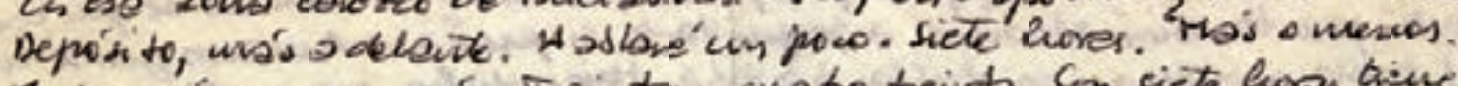
1 después nurco urs. Treinta y custro treinta. Gen siete horas bive

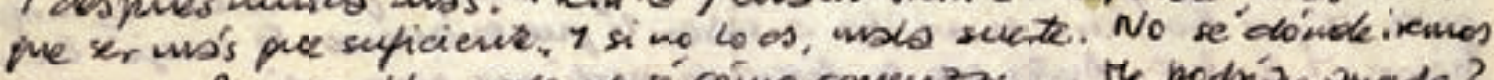

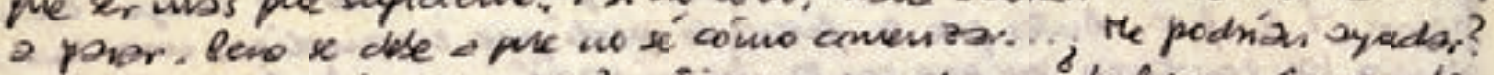

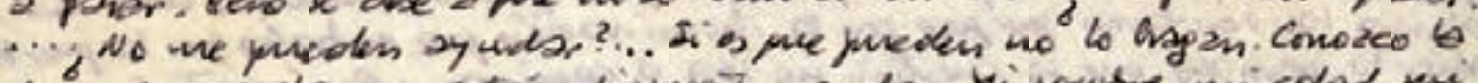

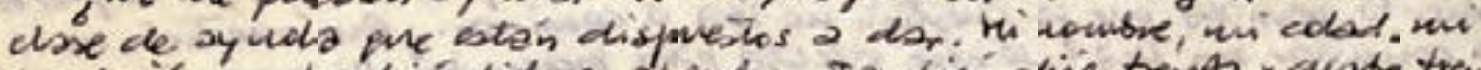

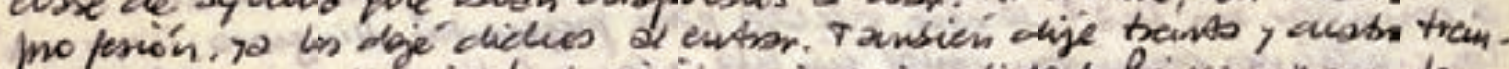

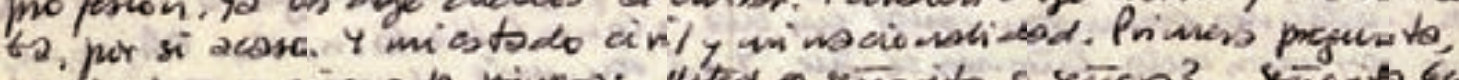

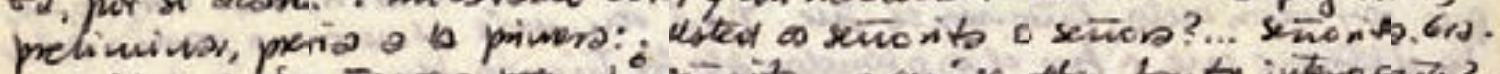

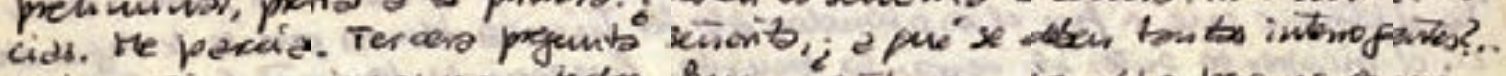

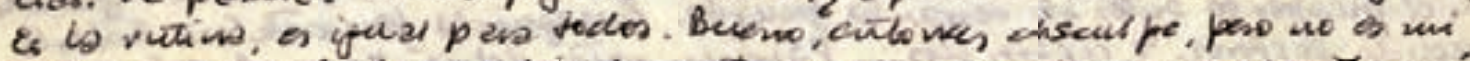

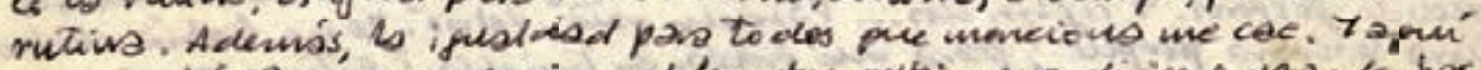
we corde! fue cuando rivo el lumbre rubio que dinige o unarda bar.

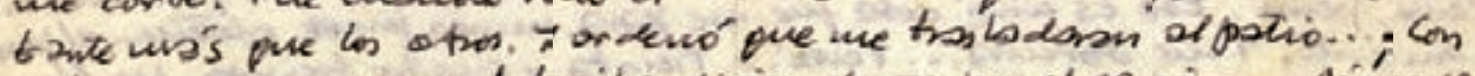

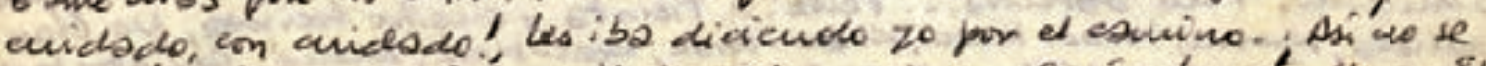

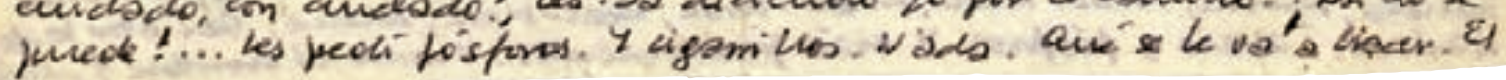




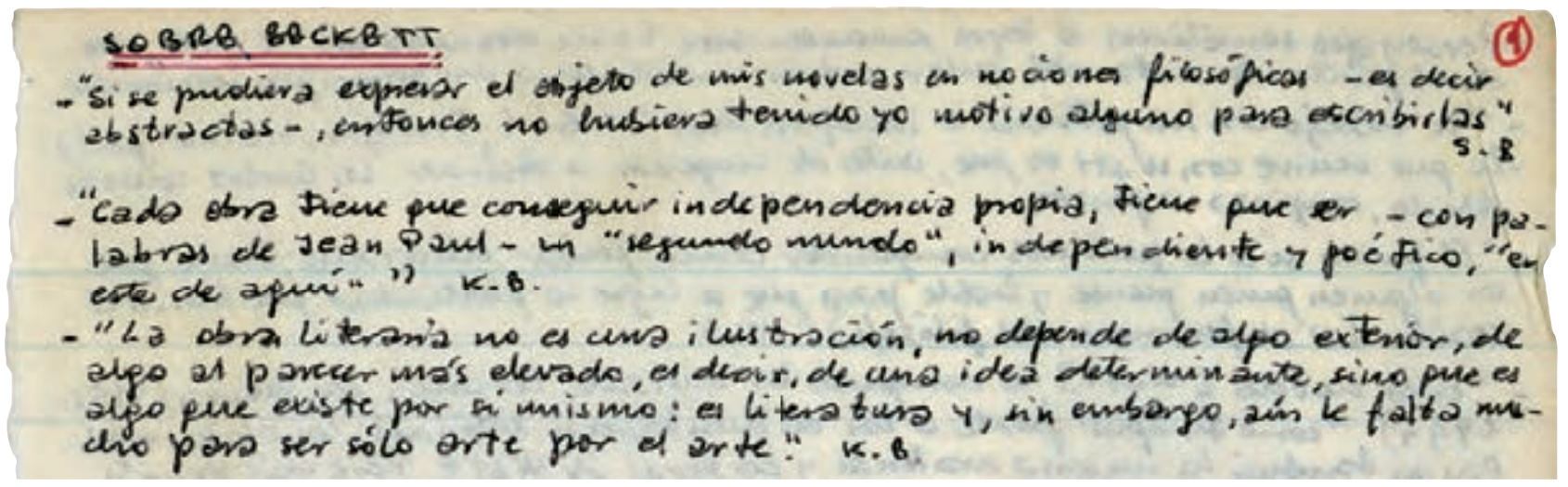

Fig. 2 : Apuntes, fol. $1 r$, archives personnelles de l'auteur, Montevideo, Uruguay.

jusqu'à 78 titres ${ }^{17}$, que les militaires contrôlaient ensuite. Après examen, le prisonnier pouvait recevoir un ou plusieurs livres dans un délai incertain, selon la disponibilité de ceuxci et des militaires.

N'étant jamais certain de pouvoir relire les ouvrages découverts, Carlos Liscano a donc constitué son propre répertoire de citations à l'aide de listes de notes de lecture dont témoignent Apuntes de la cárcel [Notes de prison]. Ce manuscrit de quatre-vingt-dix pages (quarante-cinq feuillets) est exclusivement composé de listes de citations qui rendent compte des lectures de leur auteur et qui permettent de reconstituer la bibliothèque de l'écrivain et, par extension, de la prison.

La liste est organisée, assez systématiquement, de la façon suivante : une citation d'une ou plusieurs lignes est suivie de la référence de l'œuvre, du nom de son auteur ou de ses initiales et d'une date (voir fig. 2 et 3 ). Un long trait horizontal ou un espace vient ensuite marquer la transition d'une citation, d'un auteur ou d'un sujet, vers un(e) autre. Parfois, Carlos Liscano a pu oublier une partie des informations mais ces oublis sont peu fréquents car l'écrivain a tenté, en raison des contraintes situationnelles décrites précédemment, de garder trace de ces informations bibliographiques essentielles.

Selon le sujet, Carlos Liscano a également organisé des regroupements autour d'auteurs, comme Samuel Beckett («Sobre Beckett», voir fig. 2) ou Hegel, ou de notions et thématiques telles que le langage ( $« E N G U A J E »$, voir fig. 4).

Cette liste de citations, qui semble avoir été élaborée au fil des lectures et probablement dans l'ordre des notes, apporte des informations exogénétiques fondamentales sur les sources d'inspiration de Carlos Liscano. La longueur des extraits et leur nombre permettent d'étayer certaines hypothèses, souvent signalées par la critique liscanienne, sur les références intertextuelles de ses écrits, et notamment sur l'influence de Samuel Beckett, dont on peut ici mesurer l'importance en calculant le nombre de pages ou d'extraits tirés de Molloy, En attendant Godot ou Fin de partie ${ }^{18}$. Avec vingt-deux fragments recopiés, Molloy semble, incontestablement, avoir marqué Carlos Liscano ${ }^{19}$. Nonobstant, il s'agit aussi bien de fragments tirés des œuvres de Samuel Beckett que de travaux sur lui, comme le montrent les citations issues du livre du critique allemand Klaus Birkenhauer.

Carlos Liscano a également accordé un intérêt certain aux textes sur la philosophie comme l'illustrent des citations tirées de réflexions du philosophe Hegel sur l'humour (folios $\mathrm{n}^{\circ} 14$ à 22) et aux travaux sur le langage (folios $n^{\circ} 8$ et 9 ) avec des fragments tirés d'ouvrages d'Émile Benveniste (p. 33 à 37), des citations sur la communication (folios n ${ }^{\circ} 37$ à 48) et des extraits de Problèmes de linguistique générale (folios nº 61 à 65).

L'autre caractéristique des Apuntes est que Liscano y a clairement identifié les écrivains qu'il a le plus lus, souvent des Prix Nobel de littérature : Hermann Hesse (folios $n^{\circ} 29$ à 31), Thomas Mann et Samuel Beckett, dont nous avons déjà parlé précédemment.

Dans les cas d'Hermann Hesse et de Thomas Mann, abondamment cités, nous pouvons remarquer que la lecture de ces deux auteurs a probablement suscité l'intérêt de Carlos Liscano pour le romantisme allemand comme en témoigne une autre liste dans la liste (fig. 5 et 6) : celle de dix-neuf noms d'auteurs de la période, extraite de la Antología del Romanticismo alemán, d'Antonio Mari Muñoz (1979). Il s'agit d'une liste qui n'a, bien sûr, pas vocation à être utilisée dans l'immédiat (à la fois en raison des contraintes

17. Des exemples de ces fiches figurent dans le catalogue de cette bibliothèque, publié en juillet 2013 : Alfredo Alzugarat, El Libro de los libros. Catálogo de la Biblioteca Central del Penal Libertad (1973-1985), Montevideo, Ediciones de la Biblioteca nacional del Uruguay, 2013, p. 256-259.

18. La présence des œuvres dans le catalogue de la bibliothèque de la prison a pu être vérifiée par nos soins.

19. Voir Carlos Liscano, Le Rapporteur et autres récits, Paris, Éditions 10/18, 2005. 


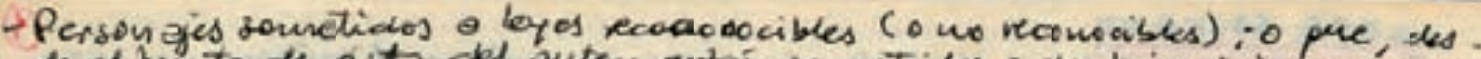

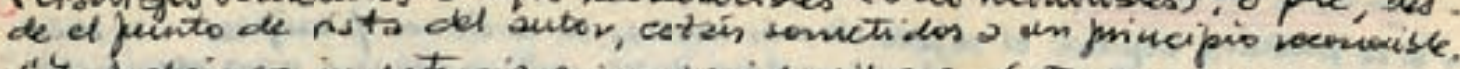

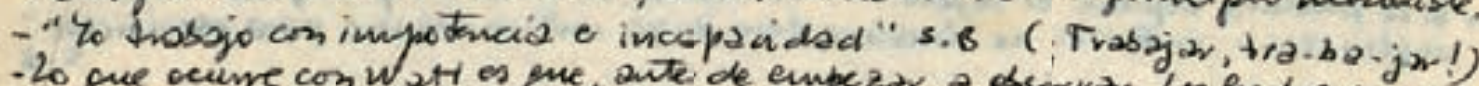
- Lo pue ocurre con Watt es pue, ante de empezar a aservar los evelos couno as desido, emprieza a parsor.

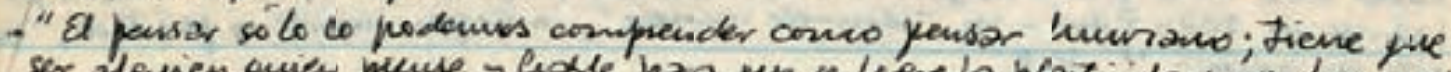

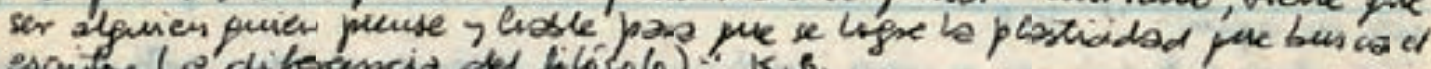
escontar (o'difenancio del filósofo) - K. 6.

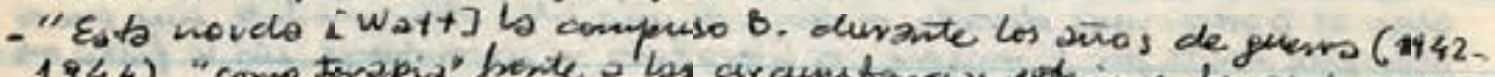

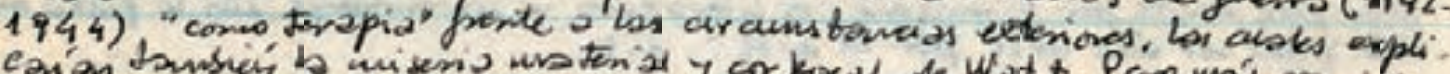

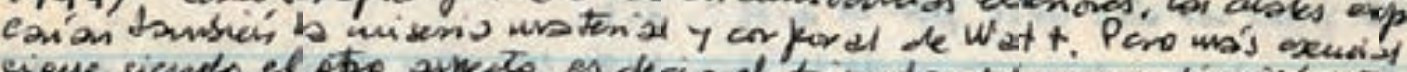

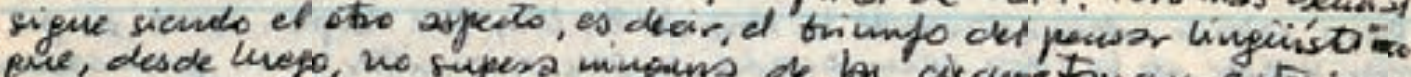

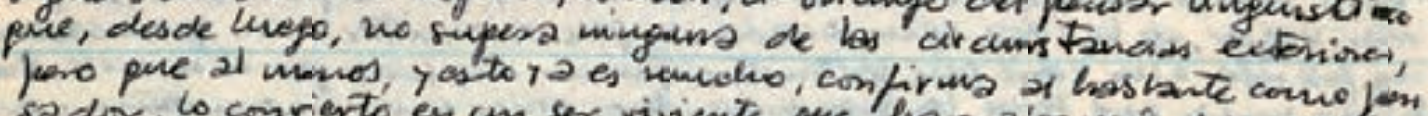
sador, lo conrierto en un ser viviente pre liece ajpo uss pre vegeter,

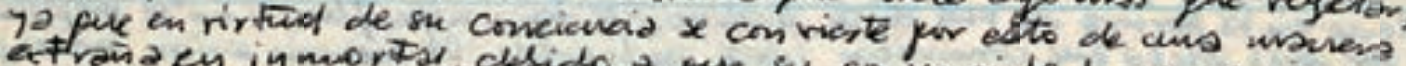
ningín $p$ apel.

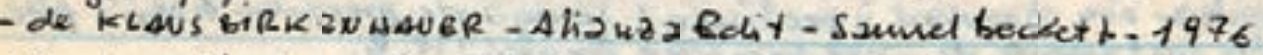

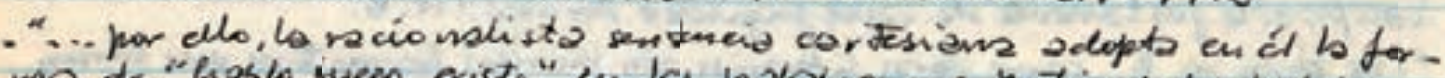
wis de "lioslo, nugo ecisto" un las palabras 72 patir de los pabsias,

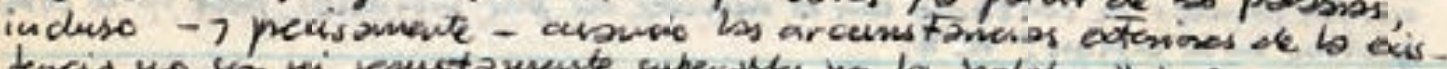

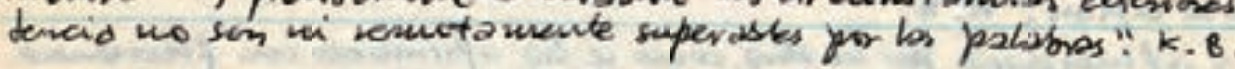

- En lar 2 primeras novelas de la tri lo gúa b. opera deniendo en cuesta

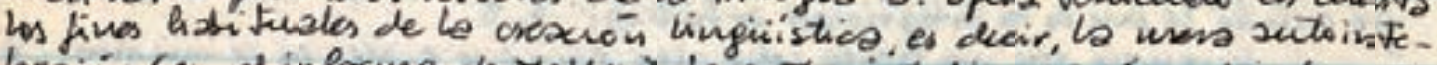

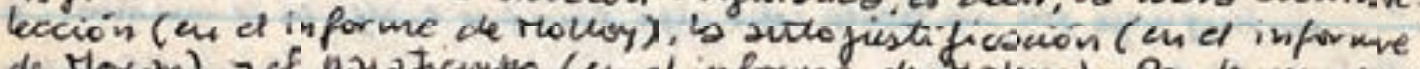

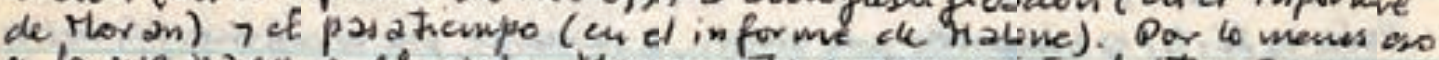

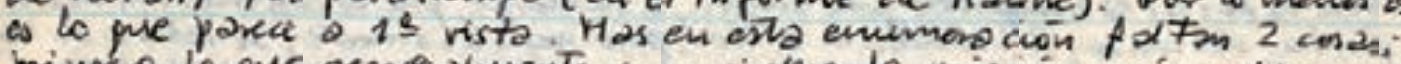

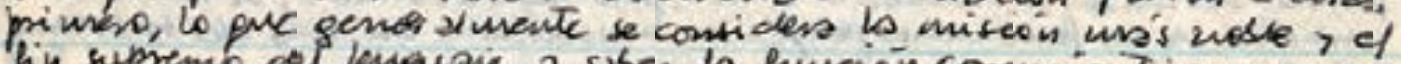

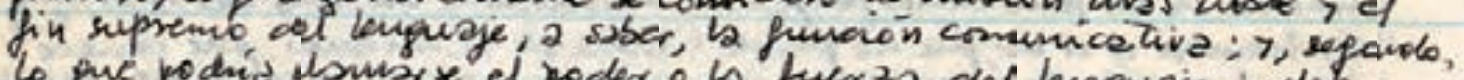

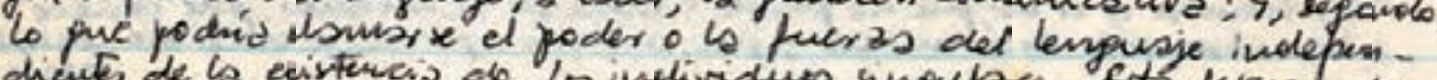

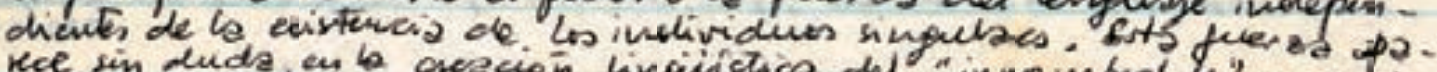
kee, sin duda, an la oncesion lingilistios del "inno inbrable" mses ipe.

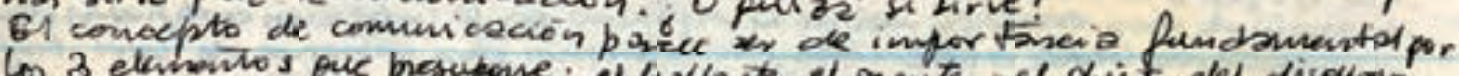

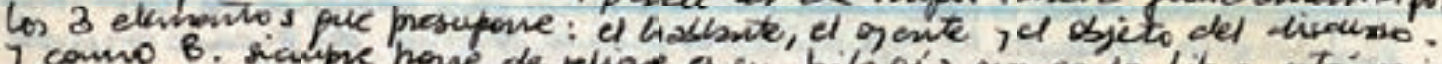

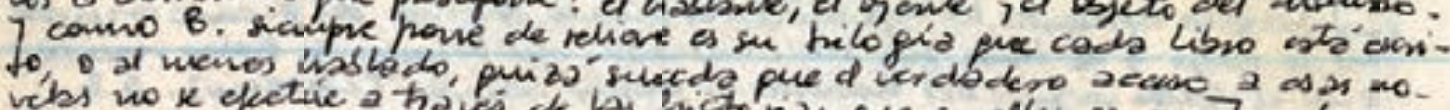

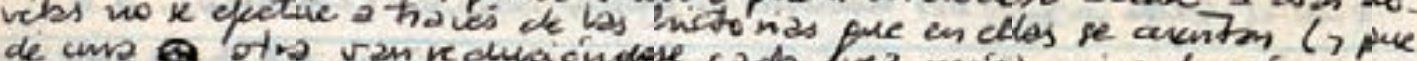

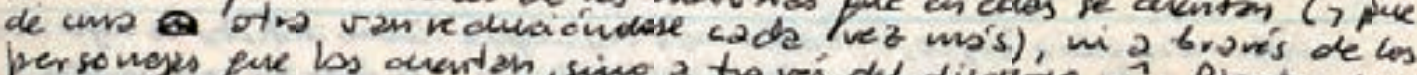

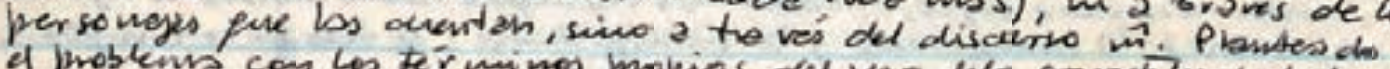

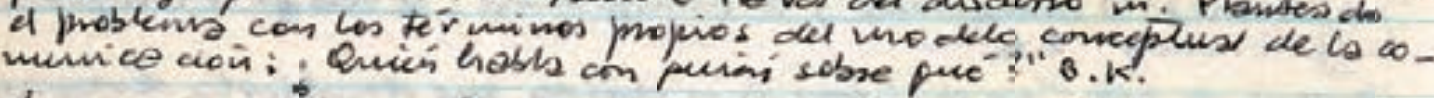

- Lo pue imports a sólo el proceso de creación lingiivitios, wo lo pue te dice; bs pstabras son sush duises e inten cambiables y tanto vitilos convo

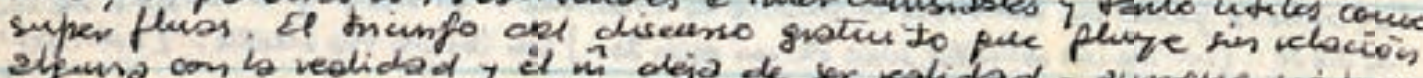

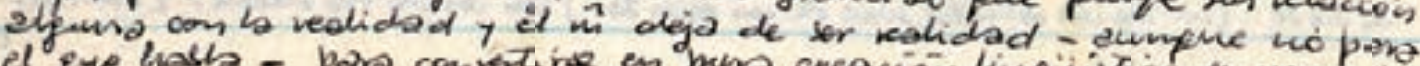

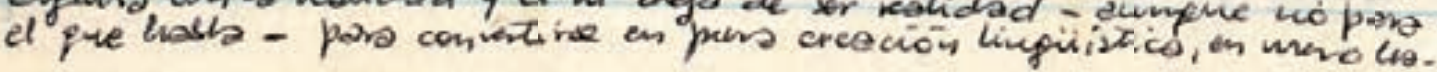




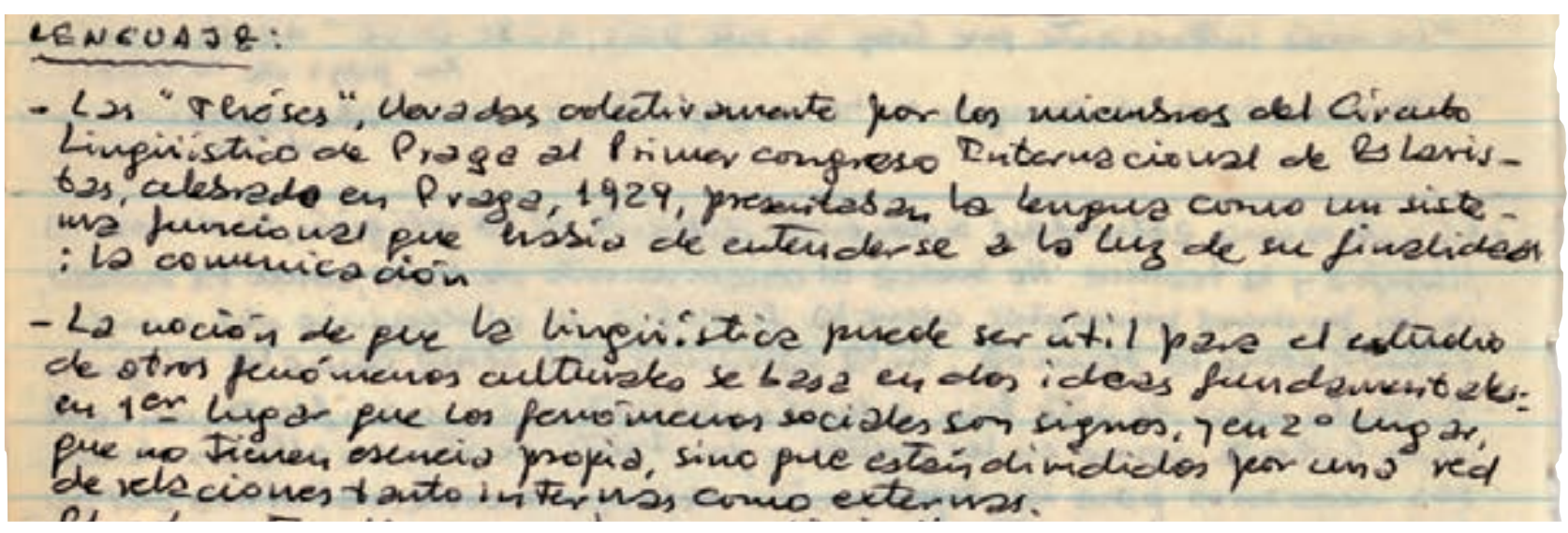

Fig. 4 : Apuntes, fol. $1 r$, archives personnelles de l'auteur, Montevideo, Uruguay.

carcérales mais également de l'indisponibilité des œuvres à la bibliothèque de la prison) mais d'un à-valoir pour l'avenir, après une éventuelle sortie de prison.

Dans les nombreux témoignages qu'il a donnés à la presse, Carlos Liscano a revendiqué l'influence des auteurs qui figurent sur ces listes : «J'ai passé ma vie à essayer d'écrire des livres qui ressemblent aux livres que j'admire ${ }^{20}$.» Ces citations, minutieusement collectées dans des conditions difficiles, pendant huit années, attestent d'un processus de formation à l'écriture qui est passé par la lecture et l'imitation du geste d'écriture littéraire. Dans le même temps, ces listes permettent d'observer les phénomènes qui précèdent le processus endogénétique, avant l'assimilation qui en efface toutes les traces. Examinons le cas de Samuel Beckett : Liscano repère dans ses écrits la force du style absurde, la vigueur du personnage solitaire, en crise, et les formes du monologue. Molloy, comme indiqué plus tôt, l'a tout particulièrement inspiré pour l'écriture de deux textes importants : le roman La Mansión del tirano [La Maison du tyran] et la nouvelle intitulée El Informante [Le Rapporteur], deux textes qui parlent du monde carcéral.

Dans La Mansión del tirano, la genèse du nom du personnage principal confirme le rôle de la liste comme étape dans un processus de formation à l'écriture qui imite d'abord. Ainsi, le personnage principal de La Mansión, appelé «Franz» dans un premier temps, voit son prénom évoluer au fil des pages du manuscrit : rebaptisé «Hans», son prénom est encore modifié pour devenir «M.». Si les prénoms Franz et Hans sont des emprunts à Franz Kafka et à Thomas Mann ${ }^{21}$, une phrase du manuscrit confirme toutefois que c'est bien Samuel Beckett qui est en filigrane 22 car Liscano calque littéralement les premières lignes de Molloy : «M. ahora soy yo» [M. maintenant, c'est moi] ${ }^{23}$. Finalement, s'il ne retient pas la phrase dans la version publiée de La Mansión del tirano, il conserve le prénom du personnage, $« \mathrm{M} »$.
En 1982, Carlos Liscano écrit dans son journal de prison qu'il lit Beckett avec un intérêt particulier, celui de pousser «plus loin» son style, revendiquant ainsi non pas l'incorporation, mais le processus endogénétique qui accompagne sa lecture du dramaturge. C'est pourquoi il lui emprunte une série de motifs comme l'idée du rapport à écrire 24 , le prénom du personnage de La Route d'Ithaque ${ }^{25}$, Vladimir ${ }^{26}$, ou des objets comme la bicyclette ou le chapeau 27 . Ainsi que l'a signalé Dirk Van Hulle, au sujet de la bibliothèque personnelle de Samuel Beckett, la lecture isole et ouvre dans le même temps l'écrivain sur une époque 28 : le manuscrit Apuntes est plus qu'une liste de références, c'est le plan de la bibliothèque d'un auteur qui, depuis sa cellule, a pu traverser les époques et les continents grâce à Balzac, Beckett, Borges, Buzzati, Céline, Cervantés, Espinola, Faulkner, Flaubert, Felisberto Hernández, Hermann Hesse, Homère, Joyce, Kafka, Mann ou Melville. Les listes de citations que

20. Carlos Liscano, Le Lecteur inconstant suivi de Vie du corbeau blanc, Paris, Belfond, 2011 ; traduction de Jean-Marie Saint-Lu y Martine Breuer, p. 17.

21. Voir Carlos Liscano, Manuscritos de la cárcel, Fatiha Idmhand (dir.), Montevideo, Ed. Caballo perdido, 2010.

22. De nombreux personnages de Beckett portent des prénoms en « $\mathbf{M} »$ : Molloy, Moran, Murphy et Malone, par exemple.

23. Samuel Beckett, Molloy, Paris, Éditions de Minuit, 2008 (1re éd. 1951). 24. Dans El Informante [Le Rapporteur]. Voir «Mon rapport sera long. Je ne l'achèverai peut-être pas », Samuel Beckett, Molloy, op. cit., p. 125. 25. Carlos Liscano, El Camino a Ítaca, Barcelone, Montesinos, 2000 (1re éd. 1994). Traduit par Jean-Marie Saint-Lu en 2005 : Carlos Liscano, La Route d'Ithaque, Paris, Belfond, 2005.

26. Samuel Beckett, En attendant Godot, Paris, Éditions de Minuit, 1952 (1re éd.).

27. Ibid.

28. «While reading is of course an isolated activity, the books in the library embed Beckett in the cultural milieu of his time», dans Mark Nixon et Dirk Van Hulle, Samuel Beckett's library, New York, Cambridge Universiy Press, 2013, p. XVI. 

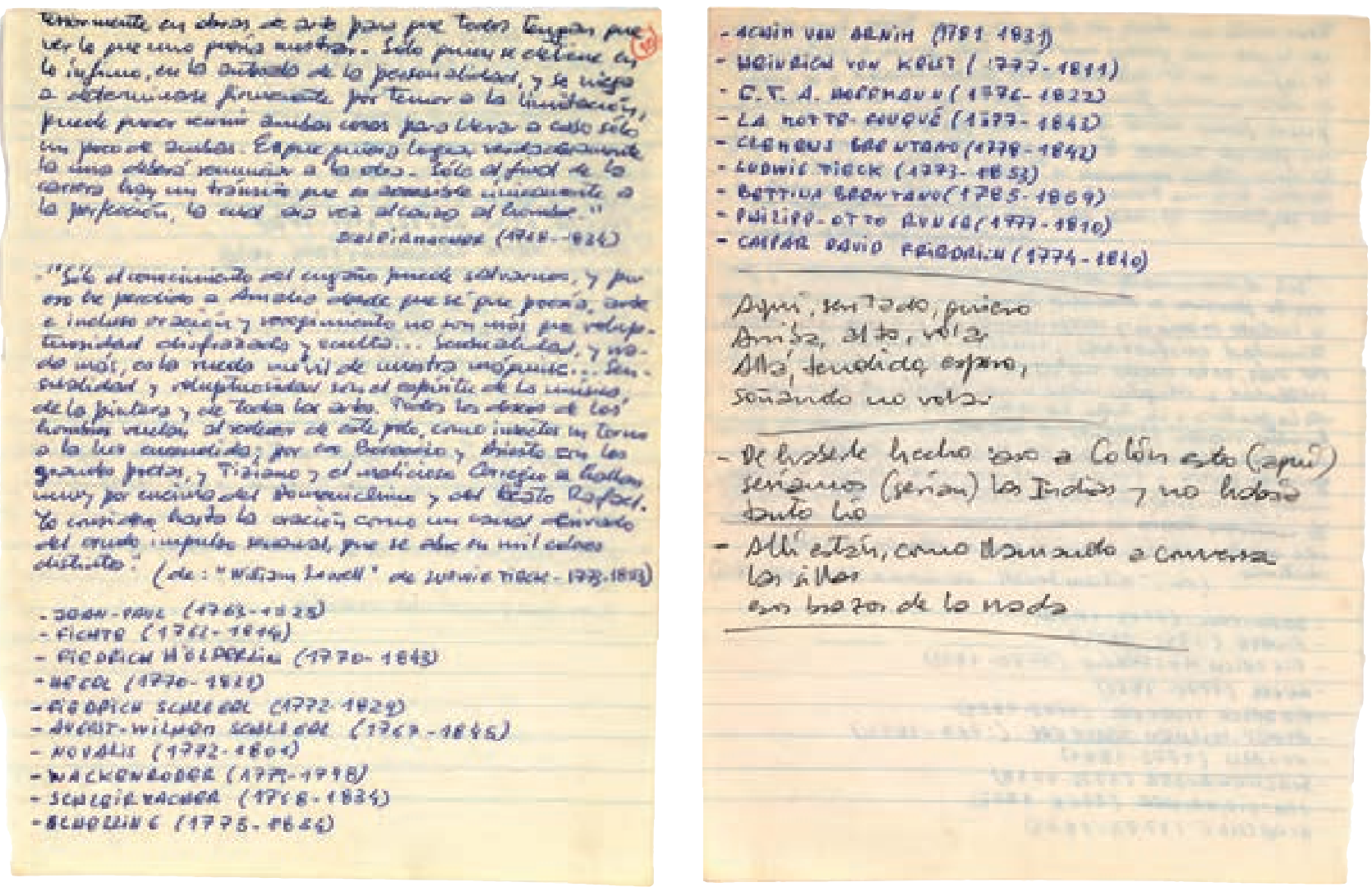

Fig. 5 et Fig. 6 : Apuntes, fol. $30 r$ et $30 v$, archives personnelles de l'auteur, Montevideo, Uruguay.

l'écrivain consulte et utilise encore, illustrent une formation née du double mouvement de lecture-écriture, de l'art de copier, d'extraire et de capturer le fonctionnement de l'écriture de ses modèles 29.

\section{Des listes de projets, des objectifs pour toute la vie}

Alors que les listes de citations ont pour but d'accompagner Carlos Liscano dans sa formation à l'écriture, par l'imitation, une autre liste se révèle déterminante dans les réalisations postérieures à l'emprisonnement : la liste de projets d'écriture.

Insérée dans le manuscrit du Diario de la Cárcel [Journal de prison] ${ }^{30}$, véritable outil de régie et de programmation ${ }^{31}$, cette liste a été rédigée en août 1983 (voir fig. 7 et 8). Elle comporte cinquante et un projets d'écriture, que Carlos Liscano introduit ainsi :

18.8 .83

Toujours là [...]. Je construis une liste de projets, deux raisons me poussent à le faire d'abord, laisser une trace de ce sur quoi je travaillais ces années-là - il y a un an, un an et demi - sur ce qu'il y avait autour, Deuxièmement : la curiosité que je pourrais avoir pour ceci à l'avenir, peut-être qu'un jour le sujet me plaira encore et que je réaliserai le projet [...]

29. La distinction entre extracteurs et marginalistes a été introduite par Daniel Ferrer («Un imperceptible trait de gomme de tragacanthe», dans Bibliothèques d'écrivains, P. D'Iorio et D. Ferrer (dir.), Paris, CNRS Éditions, 2001, p. 7-21 : p. 20) et développée par Andrei Minzetanu dans différents travaux, notamment «La lecture citationnelle ou l'ars legendi comme ars excerpendi », Littérature $\mathrm{n}^{\circ}$ 168, «Arts de lecture», 2012/4. Pour Minzetanu, les «extracteurs» (Winckelmann, Joubert, Cioran, etc.) sont des écrivains qui lisent tout en prélevant des citations pour les consigner dans leurs cahiers ou sur d'autres supports. Les «marginalistes » ou annotateurs (Voltaire, Coleridge) sont ceux qui préfèrent marquer des notes directement en marges des livres. Voir également Andrei Minzetanu, Carnets de lecture, Généalogie d'une pratique littéraire, Paris, Presses universitaires de Vincennes, 2016; Daniel Ferrer et Andrei Minzetanu, «Dialogue à propos de Carnets de lecture», Genesis, ${ }^{\circ} 43$ «Bande dessinée », 2016.

30. Ce manuscrit est fascinant par la quantité de «promesses et projets d'œuvre». À propos du rôle du journal dans le processus créatif, voir Françoise Simonet-Tenant, «Le journal comme pièce du dossier génétique », Genesis, n 32 «ournaux personnels», p. 18.

31. Voir «Le journal personnel, objet de la critique génétique? », ibid., p. 9. 

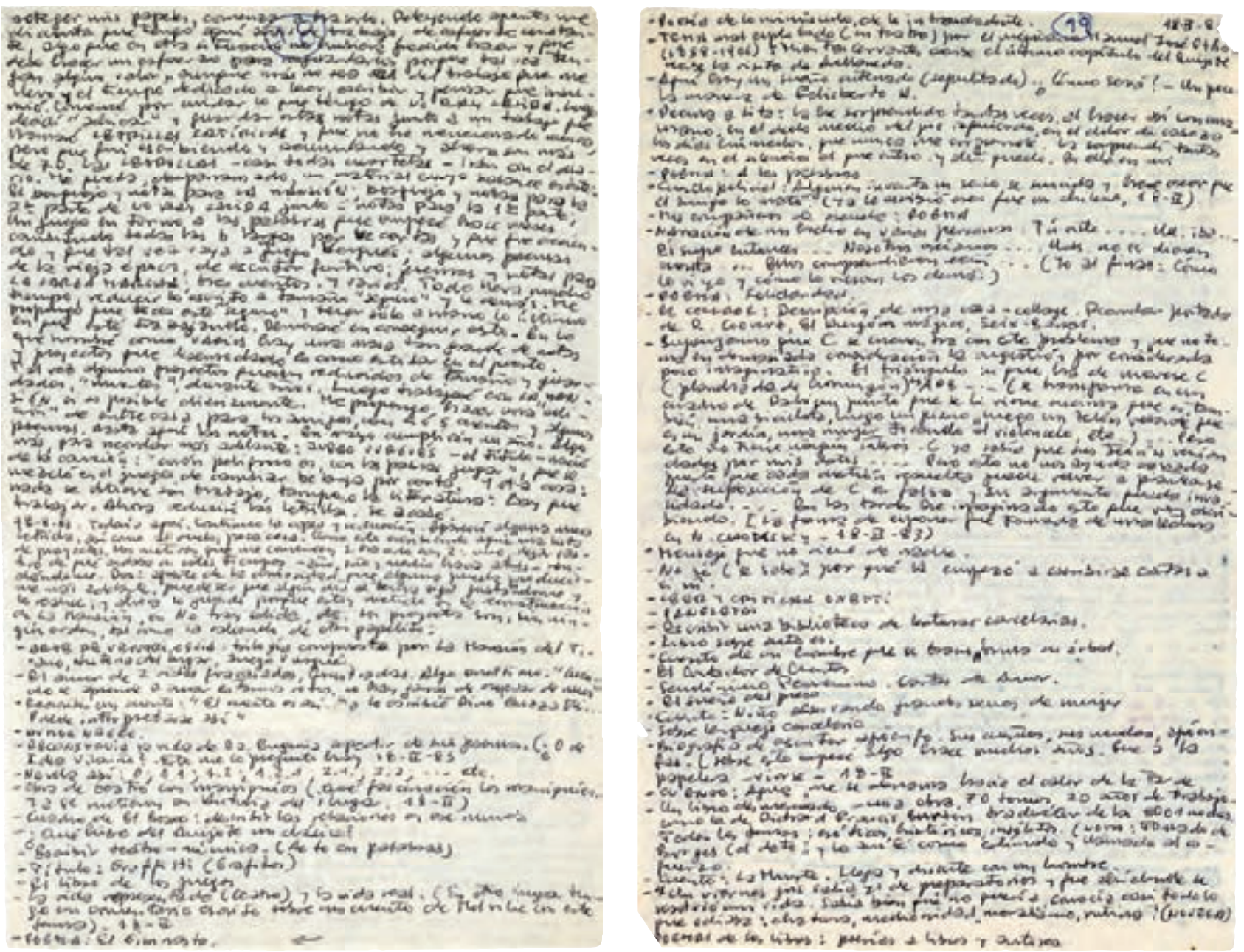

Fig. 7 et 8 : Diario de la cárcel, archives personnelles de l'auteur, Montevideo, Uruguay.

La fonction de cette liste est clairement identifiée ici : elle vise à garder une trace, à mettre en réserve des idées pour plus tard à un moment où, en 1983, rien ne lui permet d'envisager l'imminence d'une sortie de prison.

L'examen du devenir de certaines propositions de cette liste confirme son rôle prospectif : elle fixe des objectifs à très long terme, pour les mois et années à venir et il est intéressant de constater qu'à ce jour, plus de trente ans après son élaboration, Carlos Liscano continue de mettre en œuvre ce programme.

Parmi les cinquante et une propositions, nombre d'entre elles visent à raconter l'univers carcéral sous des formes diverses :

18 - Écrire une bibliothèque de lectures carcérales.

23 - Le rêve du prisonnier.

25 - Sur la langue en prison.

35 - Le style du Journal d'un escargot permettrait - hors

d'ici - d'écrire un roman sur la prison.
Journal de prison comporte ainsi le projet d'un nouveau journal $\left(\mathrm{n}^{\circ} 35\right)$ qui serait inspiré du Journal d'un escargot de Günter Grass. Le catalogue de la bibliothèque du Penal comporte quinze titres de journaux, personnels ou fictionnels, que Carlos Liscano confirme avoir lus ${ }^{32}$, et plus particulièrement ceux-ci : Journal de la guerre au cochon d'Adolfo Bioy Casares (1969), Voyage d'un naturaliste autour du monde fait à bord du navire le «Beagle» de 1831-1836 de Charles Darwin, Journal d'Anne Frank d'Anne Frank (1947), Journal d'un escargot de Günter Grass (1974), Diario de un viaje de Montevideo a Paysandú, et Diario de un Sinverguenza de Felisberto Hernández (1957), Diario de un viaje de Montevideo a Paysandú de Dámaso A. Larrañaga (1815), Journal du Doktor Faustus de Thomas Mann (1947), Journal I et II (1931-1934; 1934-1939) d'Anaïs Nin, Journal d'un fou et

32. Courrier électronique du 19 juillet 2014. 
autres nouvelles de Lu Xun 33 et Diario Íntimo de Miguel de Unamuno (1897).

Ces journaux, très différents les uns des autres, représentent autant de formes d'expression diaristes (expressions politiques, naturalistes, etc.), autant d'exemples des différentes fonctions du journal (intime, de bord, autofictionnel, fictionnel, de travail...) et autant de modèles pour l'apprenti écrivain qu'est alors Carlos Liscano. Ils précisent surtout l'horizon exogénétique de cette liste. Ainsi, lorsque Liscano indique, dans le projet $\mathrm{n}^{\circ} 35$ de sa liste, qu'il veut écrire dans «le style de Günter Grass » ${ }^{34}$, « hors d'ici», un récit «sur la prison», il nous donne un indice sur deux éléments qu'il repère dans le Journal d'un escargot : d'un côté, un personnage, Scheub, ancien SS, et de l'autre, son trait psychologique, Scheub comme figure du bourreau qui se repentit avant de se donner la mort. Ces deux éléments, consignés dans la liste sous forme d'indice (dans le «style de»), seront ensuite développés dans deux œuvres importantes de Carlos Liscano :

- la première est la nouvelle El Informante [Le Rapporteur], dont il commence la rédaction en prison (manuscrit intitulé La Edad de la prosa) et dans laquelle il raconte l'histoire d'un personnage, «je», enfermé dans une cellule et obligé de rédiger un rapport sur des faits qu'il ignore;

- l'autre est le récit-témoignage que Carlos Liscano publie en 2008 et dans lequel il raconte la torture en prison : El Furgón de los locos [Le Fourgon des fous] ${ }^{35}$. Dans ce récit, il s'intéresse à la psychologie de ses bourreaux. À ces deux œuvres, on peut ajouter le roman Vie $d u$ corbeau blanc $^{36}$, publié en 2011 d'abord dans sa traduction française. Ce roman revisite les Mille et une nuits avec, à la place de Shéhérazade, la figure oxymorique du «corbeau» blanc qui tente de survivre (car il est rejeté et poursuivi par les corbeaux noirs) en racontant des histoires. Comme dans le Journal d'un escargot, le narrateur est un animal et le style burlesque, grotesque, ainsi que les jeux de mots servent à dénoncer la rhétorique militaire et les persécutions.

Concernant le projet $\mathrm{n}^{\circ} 29$ de la liste, «écrire une bibliothèque carcérale», celui-ci voit le jour entre 2011 et 2013 sous trois formes :

- la première est le roman Vie du corbeau blanc qui comporte une série de références extra-textuelles et qui rend hommage à la puissance libératrice de la lecture en prison;
- la deuxième est le prologue intitulé Éloge du plagiat qui accompagne le recueil de récits publiés en 2013 sous le titre Fonction : ventriloque 37 qui est, comme l'indique María Carolina Blixen, une «humble reconnaissance 38 » de l'immense valeur des auteurs en prison;

- la dernière prend la forme, en 2013, de la publication, sous forme de fac-similé, du catalogue original de la bibliothèque de la prison ${ }^{39}$. Liscano pilote la publication de ce volume intitulé El Libro de los libros ${ }^{40}$.

La mise en œuvre de ces trois projets de publication, étroitement liés à la bibliothèque de la prison, coïncide avec la période durant laquelle Carlos Liscano dirige la Bibliothèque nationale de l'Uruguay (2010 à 2015). Est-ce le poste qui lui permet de donner enfin forme à ses projets de la liste?

Pour conclure, citons deux projets qui permettent de confirmer le lien qu'entretiennent les deux listes examinées dans ces quelques pages : la liste des citations et la liste des projets.

Observons d'abord les projets $n^{\circ} 36$ «a langue de la prison» et $n^{\circ} 4$ «HUMOR NEGRO» : ces deux titres confirment l'intérêt de Carlos Liscano pour la langue et son fonctionnement ainsi que nous avons indiqué plus haut. Le projet sur «la langue de la prison» a pu être

33. Lu Xun, ou Zhou Shuren (1881-1936).

34. Grass reçut le prix Nobel de littérature en 1999, bien après la sortie de prison de Carlos Liscano, en 1985.

35. Carlos Liscano, El Furgón de los locos, op. cit. Une traduction anglaise a également été publiée.

36. Carlos Liscano, Le Lecteur inconstant, op. cit.

37. Carlos Liscano, Oficio de ventriloquía 2. Relatos 1981-2011, Montevideo, Planeta, 2011, p. 48.

38. María Carolina Blixen, Carlos Liscano, Jorge Luis Borges y Macedonio Fernández : un triángulo de dos orillas, Cuadernos Lirico, $\mathrm{n}^{\circ} 8,2013$.

39. Le fac-similé a été réalisé à partir d'une copie que le poète Miguel Ángel Olivera (Montevideo, 1943-) avait conservée et que Rodolfo Wolf (ami et compagnon de cellule de Liscano) avait photocopiée. D'anciens détenus se sont mobilisés autour de ce projet de mémoire de la bibliothèque du Penal.

40. Le projet a été mis en œuvre par Alfredo Alzugarat (Montevideo, 1952-). Lui aussi a connu la prison du Penal et a utilisé le catalogue. Voir supra, note 17. 
engagé durant l'incarcération, lorsqu'il écrit La Mansión del tirano, La Edad de la prosa et El Método y otros juguetes carcelarios. Dans ces textes, il réalise un travail important d'analyse critique des formes de la subversion et de mise en forme de son style tout en imitant, nous l'avons dit, les autres écrivains. Hors de la prison, il poursuit ce travail sur la langue en travaillant l'ironie et l'humour. Le roman El Camino a Ítaca [Sur la route d'Ithaque] et les bandes dessinées intitulées Es al ñudo empezar [C'est un début] et Nulle dies sine linea [Pas un jour sans une ligne] confirment la continuité de cette réflexion. Sarcasme et humour noir y sont utilisés comme des formes d'expressions audacieuses destinées à mettre en exergue les contradictions du monde : après avoir scruté celles de la prison, Carlos Liscano examine celles de la société, ses absurdités et contrastes.

Observons pour terminer le devenir d'un autre projet, celui qui figure en première position sur la liste, «Arte de ventriloquía ». En 1984, Carlos Liscano écrit dans le journal de prison:

9.3.84 [...]

Aujourd'hui

J'ai défini mon plan pour 84 : demain je commence à écrire la trilogie Art

de la Ventriloquie

Que va-t-il en sortir? Je ne sais pas, mais la seule façon de le savoir

est de me lancer, de me mettre au

travail.

En 1984, Arte de ventriloquía devait donc prendre la forme d'une trilogie rassemblant La Mansión del tirano, Historia del lugar [Histoire du lieu] et Juego vurgués [Jeu B/Vourgeois]. Carlos Liscano ne réalisera pas le projet durant son internement mais ne l'abandonnera pas car l'idée d'écrire Arte de ventriloquía aboutira en 2011, sous le titre Oficio de ventriloquía [Fonction : ventriloque] ${ }^{41}$. Contrairement à ce qui était prévu, la forme finalement retenue ne sera pas la trilogie mais le recueil de nouvelles en deux tomes. Rien ne nous interdit de penser qu'un tome III verra le jour et que le projet de 1984 sera réalisé car dans la préface d'Oficio de ventriloquía (2011), qu'il intitule «El libro que falta» [Le livre qui manque] ${ }^{42}$, Liscano suggère déjà qu'un troisième volume est en préparation.

Comme l'a signalé Alfredo Alzugarat, la valeur des manuscrits de prison de Carlos Liscano est immense tant ils importent dans la trajectoire de l'écrivain : Liscano y revient «constamment 43 », faisant des listes de lectures et des listes de projets des sources permanentes de création. L'écrivain peut y puiser de quoi alimenter un titre, de quoi construire une épigraphe 44 ou de quoi échafauder des plans et ébauches. La spécificité de ces listes réside dans le fait qu'elles intègrent toujours les dossiers génétiques des projets les plus récents de Carlos Liscano, comme si la phase pré-rédactionnelle qui fut amorcée en prison n'avait pas encore été conclue, comme si ces brouillons appartenaient à un processus provisionnel continu (lectures, recherches préliminaires et exploratoires, pré-projets, ébauches, etc.). Telle une boussole, ces listes relancent en permanence la machine à créer.

41. Carlos Liscano, Oficio de ventriloquía 1 et 2 : Relatos 1981-2011, Montevideo, Planeta, 2011.

42. Voir la préface «El libro que falta», ibid., p. 7.

43. Voir Alfredo Alzugarat, «El constante retorno », dans Manuscritos de la cárcel, op. cit., p. 29.

44. Fatiha Idmhand, «Escribir en la cárcel : una escritura "sous contrainte" », dans Manuscritos de la cárcel, op. cit., p. 22, notes 38 à 42. 
FATIHA IDMHAND est professeur en Littératures hispaniques à l'université de Poitiers et chercheur à l'ITEM. Ses travaux portent sur la typologie des processus de création des œuvres produites « sous contraintes ». Elle étudie tout particulièrement le cas des intellectuels de l'ombre, les satellites, et leur rôle dans la circulation des idées et les transferts culturels. Également impliquée dans le champ des Humanités numériques, elle publie et réalise des éditions de manuscrits et archives d'écrivains latino-américains.

fatihaidmhand@yahoo.es

\section{Carlos Liscano : les listes comme réservoirs de projets}

Dans le champ de la littérature latino-américaine produite durant les années 1970, années de dictatures pour ce continent, rares sont les manuscrits qui ont pu témoigner, depuis l'intérieur, des formes de répression à l'encontre des écrivains. Miraculeusement sauvés des geôles, ceux de l'écrivain Carlos Liscano constituent à ce titre des brouillons exceptionnels. Ils comportent de nombreuses listes qui approvisionnent toujours, plus de trente ans après leur composition, les projets d'écriture de l'écrivain. Comment définir le statut particulier occupé par ces listes « provisionnelles » qui nourrissent, non pas un projet d'écriture, mais tous les projets de la vie de l'écrivain ? C'est à cette question que propose de répondre l'étude croisée de la genèse de ces différentes listes et de leur devenir.

Latin-American literature of the 1970's, years of dictatorship for that continent, offers few manuscripts that could, from the inside, testify to the types of repression aimed at writers. Carlos Liscano's prison manuscripts have miraculously been saved, which makes them exceptional drafts. They contain numerous lists which still, thirty years later, supply the writer's writing projects. How can one define the particular status of these "provisional" lists that sustain not merely a writing project but in fact all the writer's life projects? This article intends to answer this question with the combined study of these lists' genesis and their future.

Auf dem Gebiet der lateinamerikanischen Literatur, die in den 1970er Jahren, in den Jahren der Diktatur, für diesen Kontinent produziert wurde, konnten nur wenige Manuskripte von innen her Formen der Repression gegen Schriftsteller bezeugen. Jene des Schriftstellers Carlos Liscano, die auf wundersame Weise aus den Gefängnissen gerettet wurden, stellen in dieser Eigenschaft außergewöhnliche Entwürfe dar. Sie enthalten zahlreiche Listen, die auch mehr als dreißig Jahre nach ihrer jeweiligen Zusammenstellung noch die Schreibprojekte des Schriftstellers bereichern. Wie definiert man den besonderen Status dieser Listen, die nicht nur ein Projekt des Schreibens, sondern alle Projekte des Lebens des Schriftstellers nähren? Auf diese Frage versucht die Studie zu antworten, in der sich die verschiedenen Listen in ihrer Genese und in ihrem Werden begegnen.
En el campo de la literatura latinoamericana producida durante los años 1970 -años de dictadura en ese continente- son pocos los manuscritos que han podido testimoniar desde el interior, sobre las formas de represión sufridas por los escritores. Rescatados por milagro de la cárcel, los del escritor Carlos Liscano representan, en este sentido, borradores excepcionales. Estos manuscritos comportan numerosa listas, que siguen abasteciendo, treinta años después de su composición, los proyectos de escritura del autor. ¿Cómo definir la situación particular de estas listas "provisionales" que alimentan, no un proyecto de escritura, sino todos los proyectos vitales del escritor? Es este el interrogante al que intenta dar una respuesta el presente estudio de la génesis y de la actualidad de esas listas.

No campo da literatura da América Latina produzida durante a década de 1970, anos que foram de ditadura nesse continente, são raros os manuscritos que testemunham intrinsecamente formas de repressão sobre os escritores. Miraculosamente salvos do cativeiro, os manuscritos do escritor Carlos Liscano constituem a esse título excepcionais rascunhos. Neles se encontram numerosas listas que, trinta anos mais tarde, continuam a abastecer os projectos criativos do escritor. Como definir o estatuto especial dessas listas "provisórias" que alimentam não um, mas todos os projectos da vida do escritor? É a essa pergunta que se propõe responder o estudo cruzado da génese e do aproveitamento dessas diferentes listas.

Nel campo della letteratura latinoamericana prodotta negli anni '70, anni di dittatura per il continente, rari sono i manoscritti che hanno potuto testimoniare, dall'interno, le forme di repressione contro gli scrittori. Miracolosamente salvati dalle prigioni, quelli dello scrittore Carlos Liscano costituiscono, da questo punto di vista, dei brogliacci straordinari. Essi contengono numerose liste che alimentano ancora, trent'anni dopo la loro composizione, $\mathrm{i}$ suoi progetti di scrittura. Come definire lo statuto particolare di queste liste preparatorie che nutrono non uno ma tutti i progetti della vita dello scrittore? Lo studio incrociato della genesi di queste diverse liste e della loro evoluzione si propone di rispondere a questa domanda. 